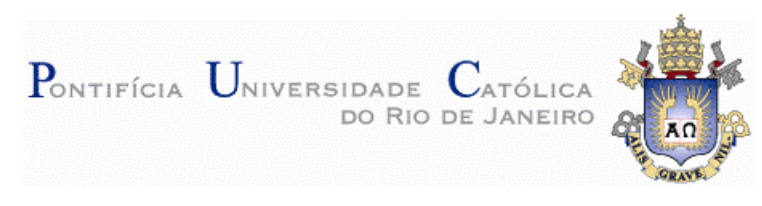

Flávia Maria Pompéia Cavalcanti

Desenvolvimento e Caracterização de um Transdutor de Campo Magnético Baseado no Efeito da Magnetoimpedância Gigante

\title{
Dissertação de Mestrado
}

Dissertação apresentada como requisito parcial para obtenção do título de Mestre pelo Programa de PósGraduação em Metrologia da PUC-Rio. Área de Concentração: Metrologia para Qualidade e Inovação.

Professores Orientadores:

Elisabeth Costa Monteiro, Ph.D.

PósMQI/PUC-Rio

Carlos Roberto Hall Barbosa, Ph.D.

PósMQI/PUC-Rio

Rio de Janeiro

Setembro de 2005 
Flávia Maria Pompéia Cavalcanti

\title{
Desenvolvimento e Caracterização de um Transdutor de Campo Magnético Baseado no Efeito da Magnetoimpedância \\ Gigante
}

Dissertação apresentada como requisito parcial para obtenção do título de Mestre pelo Programa de Pós-Graduação em Metrologia para Qualidade e Inovação da PUC-Rio. Aprovada pela Comissão Examinadora abaixo assinada.

\begin{abstract}
Elisabeth Costa Monteiro Orientador Programa de Pós-Graduação em Metrologia (PósMQI/PUC-Rio)

Carlos Roberto Hall Barbosa Co-orientador Programa de Pós-Graduação em Metrologia (PósMQI/PUC-Rio)
\end{abstract}

Fernando Luis de Araujo Machado Departamento de Física - UFPE Marcio Nogueira de Souza Departamento de Engenharia Biomédica - UFRJ

Paula Medeiros Proença de Gouvêa Programa de Pós-Graduação em Metrologia - PUC-Rio José Eugênio Leal Coordenador(a) Setorial do Centro Técnico Científico - PUC-Rio 
Todos os direitos reservados. É proibida a reprodução total ou parcial do trabalho sem autorização da universidade, da autora e do orientador.

\section{Flávia Maria Pompéia Cavalcanti}

Graduou-se em Física na Pontifícia Universidade Católica do Rio de Janeiro - PUC-Rio em 2002.

Ficha Catalográfica

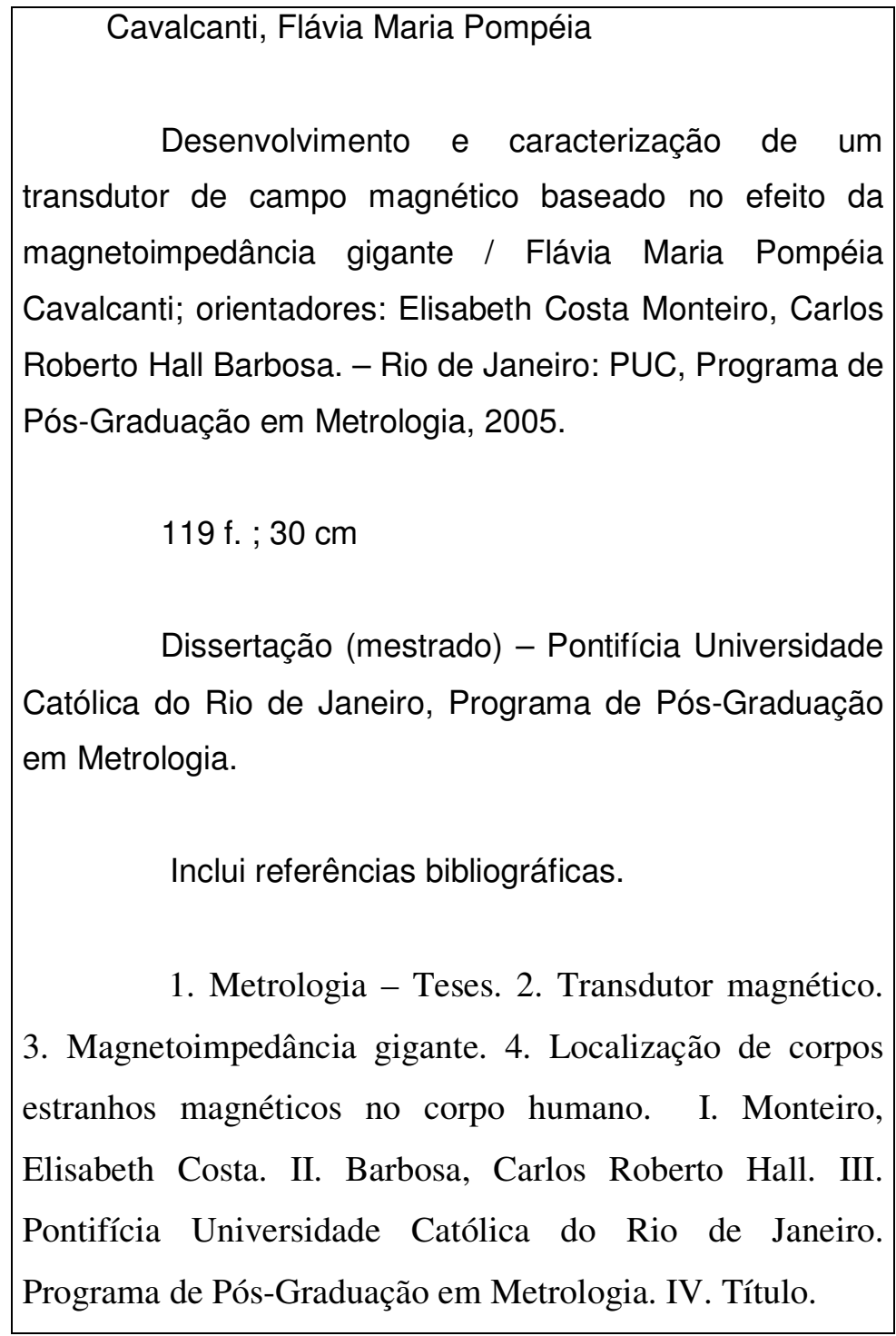

CDD: 389.1 


\section{Agradecimentos}

Ao Professor Paulo Costa Ribeiro, quem primeiro me trouxe ao mundo da física experimental e do magnetismo.

Ao Professor Daniel Acosta Avalos pelo apoio profissional e pessoal, pelo entusiasmo, por ter sempre acreditado em mim e pela amizade. Ao Professor Eduardo Chaves Montenegro, que mesmo não tendo nenhuma ligação com o projeto sempre teve as portas abertas para tirar minhas dúvidas e angústias. A ambos, pelo exemplo.

À Professora Elisabeth Costa Monteiro por ter me aceito como aluna mesmo em tempos conturbados. Pela calma e eterno otimismo.

Ao Professor Fernando Machado e toda sua equipe que me forneceram as amostras das fitas utilizadas nesta pesquisa e por terem me acolhido tão bem em seu ambiente de trabalho. Em especial à Lídice que as produziu e ao João que me ensinou mexer nos equipamentos e me acompanhou em todas as medições.

À Isabel Rios Correia e ao José Hélio Mello, sem os quais eu não estaria aqui hoje.

À minha família: Renata, Claudia, Sueli, aos Carlos, André, Pri ... e às minhas crianças, Luc e Nicolas. Aos meus pais que me acompanharam de perto durante todo este percurso e que, mesmo possuindo profissões tão diferentes, pacientemente me ouviram falar do trabalho e me deram bons conselhos. Ao meu pai por toda cobrança.

Aos meus amigos, peças fundamentais para que este trabalho pudesse ser realizado, que juntamente com minha família constituem meu mais precioso bem: Samantha, Diogo, Pedro, Renato, Renata, Geraldo... e a todos que habitam o cavernão e/ou estiveram por perto. E em especial ao Lucas, e à Clara, minha companheira de todas as horas. 
Finalmente aos Professores Carlos Roberto Hall Barbosa e Luis A. P. Gusmão sem os quais este trabalho não teria sido realizado. Ao Hall por ter me assumido, pela paciência e por ter me ajudado a não desistir nos momentos de desespero e desânimo. Ao Gusmão, meu companheiro de laboratório, por todos os circuitos e engenhocas desenvolvidas, pelo entusiasmo, pelas discussões e pelo esforço conjunto. A ambos pelo bom ambiente de trabalho.

A todos os professores do Departamento de Física e do Programa de PósGraduação em Metrologia que compartilharam comigo seus conhecimentos e contribuíram para meu crescimento acadêmico.

Ao CNPq e à PUC-Rio pelos auxílios concedidos. 


\section{Resumo}

Pompéia Cavalcanti, F. M. Desenvolvimento e Caracterização de um Transdutor de Campo Magnético Baseado no Efeito da Magnetoimpedância Gigante. Pontifícia Universidade Católica do Rio de Janeiro, 2005. 119p. Dissertação de Mestrado - Programa de Pós-Graduação em Metrologia para Qualidade e Inovação (PósMQI), Pontifícia Universidade Católica do Rio de Janeiro.

Nesta dissertação são descritos o projeto, a implementação e os testes preliminares de um protótipo de transdutor de campo magnético em tensão, baseado no fenômeno da Magnetoimpedância Gigante (MIG) e caracterizado por uma configuração geométrica inovadora. O elemento sensor e o circuito eletrônico do transdutor foram idealizados e implementados de modo a se obter a maior sensibilidade possível a campos próximos e imunidade a campos remotos. Através das medidas de caracterização do elemento sensor e do circuito, foi possível obter uma sensibilidade estimada do transdutor, sendo esta aproximadamente $12 \mathrm{~V} / \mathrm{Oe}$, a qual já é comparável à de dois dos principais sensores magnéticos existentes, o fluxgate e o sensor de efeito Hall. O transdutor desenvolvido tem por principal aplicação, nesta pesquisa, a localização de corpos estranhos magnéticos no corpo humano, baseando-se em técnica já desenvolvida e testada para sensores SQUID. Para auxiliar a interpretação dos resultados experimentais, foi criado um modelo teórico do campo magnético associado a uma agulha e do sinal de saída do transdutor associado a este campo. Medições com uma agulha retilínea foram realizadas para se avaliar o comportamento do transdutor perante este campo magnético. Apesar da elevada sensibilidade, as medidas indicaram não-linearidade e baixa imunidade a campos uniformes. Contudo, independentemente das melhorias que possam ser feitas e que já foram identificadas, o transdutor desenvolvido já tem aplicações bastante promissoras, destacando-se por seu reduzido custo de fabricação e operação.

\section{Palavras-chave}

Metrologia ; transdutor magnético; magnetoimpedância gigante; localização de corpos estranhos magnéticos no corpo humano. 


\section{Abstract}

Pompéia Cavalcanti, F. M. Development and Characterization of a Field Magnetic Transducer Based on Giant Magnetoimpedance Effect. Pontifícia Universidade Católica do Rio de Janeiro, 2005. 119p. Msc. Dissertation - Programa de Pós-Graduação em Metrologia para Qualidade e Inovação (PósMQI), Pontifícia Universidade Católica do Rio de Janeiro.

In this dissertation, it is proposed the design of a magnetic field to voltage transducer based on the Giant Magnetoimpedance phenomenon (GMI), characterized by an innovative geometric configuration. In order to attain the best near-field sensibility and far-field immunity, the transducer's sensitive element and electronic circuit were planned and implemented. By thoroughly characterizing them it was possible to obtain an estimate of the transducer's sensibility, which is approximately $12 \mathrm{~V} / \mathrm{Oe}$. This value is comparable to those observed in two of the most important existing magnetic sensors: the fluxgate and the Hall effect sensor. The main application of the developed transducer is the localization of magnetic foreign bodies in humans, based on a previously developed and tested SQUID sensor technique. In order to provide a better interpretation of the experimental results, a theoretical model of the magnetic field associated with a needle and of the signal it generates in the transducer was created. Measurements with a needle were performed to analyze the behavior of the prototype, which has a high sensitivity, as expected, but presents strong hysteresis, lack of linearity and low immunity to uniform fields. However, despite the improvements that can still be done and have already been identified, the developed transducer has many promising applications, and has the advantage of reduced fabrication and operation costs.

\section{Key-Words}

Metrology; magnetic transducer; giant magnetoimpedance; localization of magnetic foreign bodies in humans. 


\section{Sumário}

1 Introdução 20

1.1. Motivação 20

1.2. Técnica de Localização de Corpos Estranhos Magnéticos com o Sensor SQUID 22

1.2.1. Aparato Experimental 22

1.2.2. Algoritmo de Localização 23

1.3. Magnetômetros 26

1.3.1. SQUID 27

1.3.2. Sensor de Efeito Hall 29

1.3.3. Sensor Fluxgate 29

1.4. Fenômeno da Magnetoimpedância Gigante (MIG) 31

1.5. Confiabilidade Metrológica de Equipamentos Eletromédicos 32

1.6. Estrutura e Conteúdo da Dissertação 34

2 Fita MIG 36

2.1. Introdução 36

2.2. Fitas Ferromagnéticas Amorfas 38

2.2.1. Ligas Ferromagnéticas Amorfas 38

2.2.2. Produção das Fitas - Técnica Melt-Spinning 39

2.3. O Fenômeno da MIG do tipo MIL 40

2.3.1. Impedância 40

2.3.2. Descrição Física 42

2.3.3. Modelos para o Regime de Freqüências Moderadas 45

2.4. Caracterização da Fita MIG 47

2.4.1. Aparato Experimental 48

2.4.2. Curva $|Z| \times|\vec{H}|$ 
3 Transdutor MIG de Campo Magnético em Tensão 53

3.1. Elemento Sensor 53

3.1.1. Análise da Impedância para Sinais Magnéticos de Baixa Intensidade 53

3.1.2. Configuração do Elemento Sensor 54

3.1.3. Integração do Campo Magnético na Fita MIG em Forma de Anel 55

3.1.4. Medidas de Caracterização do Elemento Sensor de Campo Magnético 56

3.2. Circuito Eletrônico $\quad 59$

3.3. Transdutor Completo 61

3.3.1. Variação do Sinal de Saída do Transdutor com a Freqüência da Corrente de Alimentação das Fitas 61

3.3.2. Histerese 62

3.3.3. Rejeição a Campos Uniformes 64

4 Modelagens $\quad 68$

4.1. Campo Magnético Gerado por uma Agulha Retilínea 68

4.1.1. Modelo para o Campo Magnético de uma Agulha Retilínea 68

4.1.2. Medidas com o Sensor Fluxgate 76

4.1.3. Calibração do Modelo - Estimação do Momento Magnético da Agulha $\quad 78$

4.2. Simulação do Campo Magnético Detectado pelo Anel Sensor 80

4.2.1. Anel Sensor com Configuração Horizontal 80

4.2.2. Anel Sensor com Configuração Vertical 83

4.3. Análise da Orientação do Anel Sensor 85

4.4. Análise do Raio do Anel Sensor 87

4.5. Modelagem do Sinal de Saída do Transdutor 88

4.5.1. Mapas do Sinal de Saída 89

4.5.2. Análise Geral do Sinal de Saída 90

5 Resultados Experimentais 92

5.1. Medições Unidimensionais 93

5.1.1. Anel na Configuração Vertical 93 
5.1.2. Anel na Configuração Horizontal 101

5.2. Medições Bidimensionais 107

5.2.1. Anel na Configuração Vertical 108

5.2.2. Anel na Configuração Horizontal 109

6 Discussão, Trabalhos Futuros e Conclusão 111

6.1. Discussão 111

6.2. Trabalhos Futuros 113

6.3. Conclusão 114

$\begin{array}{lr}\text { Referências Bibliográficas } & 115\end{array}$

Anexo - Diagrama do Circuito Eletrônico do Transdutor 116 


\section{Lista de tabelas}

Tabela 1.1 - Magnetômetros, resolução e preço. 27

Tabela 2.1 - Sistema de Unidades 48

Tabela 4.1 - Valores de momento de dipolo magnético para cada altura $(h)$ do sensor ao longo do eixo $z$. 


\section{Lista de figuras}

Figura 1.1 - Arranjo experimental para localização de agulhas usando o sensor SQUID [1].

Figura 1.2 - Mapa do campo magnético detectado pelo SQUID num plano $3 \mathrm{~cm}$ acima da pele do paciente [1].

Figura 1.3 - Campo magnético normalizado gerado por uma agulha de $4,5 \mathrm{~cm}, \operatorname{com} \theta=40^{\circ}$ e $h=12 \mathrm{~cm}$.

Figura 1.4 - (a) Gráfico de $D \times R$ variando-se a profundidade do sensor em relação ao corpo estranho; (b) variando-se a inclinação da agulha em relação ao plano de medição; (c) gráfico de $D \times R$, onde cada linha corresponde a um valor de deslocamento $(\Delta)$ [1].

Figura 1.5 - Anel supercondutor interrompido por uma junção Josephson.

Figura 1.6 - Esquema do aparato do sensor SQUID.

Figura 1.7 - Representação de um sensor de efeito Hall.

Figura 1.8 - Representação de um sensor fluxgate parcialmente completo.

Figura 1.9 - Histerese.

Figura 1.10 - Representação de um sensor fluxgate.

Figura 1.11 - Dependência do módulo da impedância $Z$ com o campo magnético externo $H_{e x}$ para uma fita de ( $\left.\mathrm{Fe}{ }_{0.053} \mathrm{Co}{ }_{0.947}\right){ }_{70} \mathrm{Si}_{12} \mathrm{~B}_{18}$, com corrente de medida de $900 \mathrm{kHz}$ de freqüência e $5 \mathrm{~mA}$ de amplitude. (a) comportamento de pico único, (b) comportamento de pico duplo [21].

Figura 2.1 - Representação da técnica Melt - Spinning.

Figura 2.2 - Desenho esquemático de uma amostra em forma de fita, sendo submetida à passagem de uma corrente elétrica alternada I e na presença de um campo magnético externo. Através da medida de tensão $V$ induzida nos terminais do fio, é calculada a sua impedância $Z$. 
Figura 2.3 - Dependência do módulo da impedância $Z$ com o módulo do campo magnético externo para uma fita de ( $\left.\mathrm{Fe}{ }_{0.053} \mathrm{Co}_{0.947}\right)_{70} \mathrm{Si}_{12} \mathrm{~B}_{18}$, na faixa de freqüência mediana $(900 \mathrm{kHz})$, com corrente aplicada de $5 \mathrm{~mA}$. (a) antes de sofrer tratamento térmico, (b) depois de passar por tratamento térmico. [21]

Figura 2.4 - Ilustração da configuração dos domínios magnéticos em uma fita MIG.

Figura 2.5 - llustração da configuração dos domínios magnéticos numa fita MIG com aplicação de um campo magnético externo.

Figura 2.6 - Ilustração da configuração dos domínios magnéticos numa fita MIG com aplicação de um campo magnético externo e de um campo induzido pela corrente.

Figura 2.7 - Diagrama em blocos do sistema utilizado na caracterização das fitas MIG.

Figura 2.8 - Diagrama esquemático do par de Helmholtz.

Figura 2.9 - Curva de calibração para o par de Helmholtz utilizando a média das correntes e barras de incerteza associadas.

Figura 2.10 - Caracterização $|z| \times|\vec{H}|$ da fita MIG utilizada neste trabalho, com corrente de alimentação de $10 \mathrm{~mA}$ de amplitude e $1 \mathrm{MHz}$ de freqüência.

Figura 3.1 - Pequena variação da impedância para uma pequena variação do campo, em torno de um ponto de polarização $\left(\mathrm{Z}_{0}, \mathrm{H}_{0}\right)$.

Figura 3.2 - Protótipo do sensor : (a) fitas MIG fixadas ao anel interno; (b) com anel de proteção e as espiras para gerar o campo de polarização. 55 Figura 3.3 - Espira.

Figura 3.4 - Curva $|Z| x|\vec{H}|$ para o anel sensor, com corrente de alimentação de $10 \mathrm{~mA}$ de amplitude e $1 \mathrm{MHz}$ de freqüência.

Figura 3.5 - Curvas $|Z| x|\vec{H}|$ para a configuração retilínea da fita MIG (Zreta) e para a configuração da fita em formato de espira (Zanel).

Figura 3.6 - Curvas de histerese da relação da impedância com o campo magnético, para o anel sensor. 
Figura 3.7 - Diagrama do circuito eletrônico de alimentação e leitura. $\quad 59$ Figura 3.8 - Variação da amplitude do sinal de saída do transdutor em função do campo magnético aplicado, para diferentes freqüências da corrente de alimentação das fitas MIG.

Figura 3.9 - Amplitude máxima do sinal de saída do transdutor em função da freqüência da corrente de alimentação das fitas MIG.

Figura 3.10 - Curvas de histerese para o elemento sensor, em linha cheia, e para o transdutor em linha tracejada.

Figura 3.11 - Curvas de histerese para o transdutor.

Figura 3.12 - Diagrama esquemático do experimento da variação do sinal do transdutor com a direção do campo da Terra, (a) anel na horizontal, (b) anel na vertical.

Figura 3.13 - Variação do sinal do transdutor com a direção do campo da Terra.

Figura 3.14 - Configurações do experimento para se medir a influência de campos uniformes sobre o transdutor. (a) Plano do anel sensor paralelo ao plano das bobinas de Helmholtz; (b) Plano do anel sensor perpendicular ao plano das bobinas de Helmholtz.

Figura 3.15 - Curvas do sinal de saída do transdutor em função do campo gerado pela bobina de Helmholtz, para diferentes configurações e campos de polarização.

66

Figura 4.1 - Posicionamento da agulha na simulação (alinhada com o eixo $\mathrm{X})$.

Figura 4.2 - Agulha inclinada em relação ao plano XY.

Figura 4.3 - Projeção das componentes do campo gerado pela agulha inclinada em relação ao plano XY, no sistema de coordenadas original.

Figura 4.4 - Agulha inclinada em relação ao plano XZ.

Figura 4.5 - Projeção das componentes do campo magnético gerado pela agulha inclinada em relação ao plano $\mathrm{XZ}$, no sistema de coordenadas original. 
Figura 4.6 - Mapas simulados das componentes do campo magnético gerado por uma agulha retilínea que é representada por uma reta preta, centralizada nos mapas. (a) $B_{x}$, (b) $B_{y}$ e (c) $B_{z}$. 75

Figura 4.7 - Linhas de campo de um ímã.

Figura 4.8 - Magnetização normalizada da agulha como função do tempo de exposição [2].

Figura 4.9 - $\mathrm{Na}$ curva em azul estão representados os valores da componente $B z$ do campo magnético ao longo da linha central (eixo $\mathrm{x}$ ) onde se localiza a agulha, medido a $10 \mathrm{~cm}$ de altura. Na curva em verde estão representados os valores da componente $B z$ do campo teórico, utilizando o momento de dipolo magnético calculado.

Figura 4.10 - Diagrama esquemático para a simulação do campo magnético detectado, utilizando a configuração horizontal da espira. $\quad 81$ Figura 4.11 - Mapa simulado do campo magnético total tangencial a espira sensora na horizontal, com raio $=2,5 \mathrm{~cm}$, gerado por uma agulha alinhada com o eixo $\mathrm{X}$, com $I=4,5 \mathrm{~cm}, m=7,75 \times 10^{-4} \mathrm{Am}^{2}$ e $\theta=0$. Amplitude máxima $=2,11 \times 10^{-6}$ Oe.

Figura 4.12 - Mapa simulado do campo magnético total tangencial a espira sensora na horizontal, com raio $=2,5 \mathrm{~cm}$, gerado por uma agulha alinhada com o eixo X, com $I=4,5 \mathrm{~cm}, m=7,75 \times 10^{-4} \mathrm{Am}^{2}$ e $\theta=\pi / 6$.

Figura 4.13 - Diagrama esquemático da configuração vertical da espira, utilizado na simulação.

Figura 4.14 - Mapa simulado do campo magnético total tangencial a espira sensora na vertical, com raio $=2,5 \mathrm{~cm}$, gerado por uma agulha alinhada com o eixo $\mathrm{X}$, com $I=4,5 \mathrm{~cm}, m=7,75 \times 10^{-4} \mathrm{Am}^{2}$ e $\theta=0$. Amplitude máxima $=2,31 \times 10^{-6}$ Oe.

Figura 4.15 - Mapa simulado do campo magnético total tangencial a espira sensora na vertical, com raio $=2,5 \mathrm{~cm}$, gerado por uma agulha alinhada com o eixo $X, \operatorname{com} I=4,5 \mathrm{~cm}, m=7,75 \times 10^{-4} \mathrm{Am}^{2}$ e $\theta=\pi / 4$.

Figura 4.16 - Variação da amplitude do campo magnético com o ângulo entre a agulha e o plano $\mathrm{XY}$, considerando a altura do sensor relativa ao centro das espiras, para a espira sensora na posição horizontal (a) e vertical (b). 
Figura 4.17 - Variação da amplitude do campo magnético em função do ângulo $\varphi$ entre a agulha e 0 eixo $X$ utilizando a configuração vertical da espira.

Figura 4.18 - Amplitude máxima do campo detectado em função do raio. (a) sensor na configuração horizontal, (b) sensor na configuração vertical.

Figura 4.19 - Mapa do sinal de saída do transdutor simulado, com a espira na vertical, $\mathrm{x}_{\mathrm{c}}=\mathrm{y}_{\mathrm{c}}=(-15,15) \mathrm{cm}, h=10 \mathrm{~cm}, l=4,5 \mathrm{~cm}, \mathrm{r}=2,5 \mathrm{~cm}, m=$ $7,75 \times 10^{-4} \mathrm{Am}^{2}$ e $\alpha=3 \Omega /$ Oe. Amplitude máxima $=2,78 \times 10^{-5} \mathrm{~V}$.

Figura 4.20 - Mapa do sinal de saída do transdutor simulado, com a espira na vertical, $\mathrm{x}_{\mathrm{c}}=\mathrm{y}_{\mathrm{c}}=(-15,15) \mathrm{cm}, h=10 \mathrm{~cm}, I=4,5 \mathrm{~cm}, \mathrm{r}=2,5 \mathrm{~cm}, m=$ $7,75 \times 10^{-4} \mathrm{Am}^{2}$ e $\alpha=3 \Omega /$ Oe. Amplitude máxima $=2,54 \times 10^{-5} \mathrm{~V}$. 89 Figura 4.21 - Variação do sinal de saída do transdutor com a altura do sensor, (a) espira na configuração vertical, (b) espira na configuração horizontal.

Figura 4.22 - Variação do sinal de saída do transdutor com a intensidade do momento de dipolo magnético, (a) espira na configuração vertical, (b) espira na configuração horizontal.

Figura 5.1 - Esquema do aparato experimental utilizado para realizar as medições com a agulha.

Figura 5.2 - Diagrama do experimento em uma dimensão, (a) anel sensor na configuração vertical, (b) anel sensor na configuração horizontal. 93 Figura 5.3 - Diagrama do experimento geral com o anel na configuração vertical.

Figura 5.4 - Avaliação da repetitividade dos resultados das medições do sinal de saída em função da posição da agulha, utilizando o transdutor com o anel posicionado na configuração vertical.

95

Figura 5.5 - Diagrama esquemático do experimento para avaliar a variação do comportamento da curva da Fig. 5.4 com o sentido do movimento da agulha, utilizando o anel sensor na configuração vertical. (a) movimento da esquerda para direita, (b) da direita para esquerda. 
Figura 5.6 - Medidas do sinal de saída do transdutor com anel sensor na configuração vertical, em função da posição da agulha, para sentidos opostos de movimentação da agulha. 96

Figura 5.7 - Esquema do experimento para avaliar a variação do sinal de saída do transdutor com a posição do pólo da agulha, utilizando o anel sensor na configuração vertical. (a) pólos com sentido normal, (b) pólos girados de $180^{\circ}$.

Figura 5.8 - Medidas do sinal de saída do transdutor com anel sensor na configuração vertical, em função da posição da agulha, para sentidos opostos de posição do pólo da agulha.

97

Figura 5.9 - Esquema do experimento para analisar o comportamento do transdutor em função da direção do campo magnético da Terra, com o anel na configuração vertical. (a) anel perpendicular ao campo magnético terrestre, (b) anel paralelo ao campo magnético terrestre. 98 Figura 5.10 - Esquema do experimento para analisar o comportamento do transdutor em função da direção do campo da Terra, com anel na configuração vertical. (a) anel sensor perpendicular ao campo magnético terrestre, (b) anel sensor paralelo ao campo magnético terrestre.

Figura 5.11 - Variação do sinal de saída do transdutor com anel sensor na configuração vertical, em função da posição da agulha, para distâncias entre o sensor e a agulha de $2 \mathrm{~cm}$ a $5 \mathrm{~cm}$.

Figura 5.12 - Amplitude máxima do sinal de saída em função da distância entre o sensor e a agulha, para o anel na configuração vertical. 100 Figura 5.13 - Esquemático do experimento geral com anel na configuração horizontal.

Figura 5.14 - Avaliação da repetitividade dos resultados das medições do sinal de saída em função da posição da agulha, utilizando o transdutor com o anel posicionado na configuração horizontal.

101

Figura 5.15 - Esquema do experimento para avaliar a variação do comportamento da curva do sinal de saída com a posição da agulha, para sentidos opostos do movimento da agulha, utilizando o anel sensor na configuração horizontal: (a) movimento de ida, (b) movimento de retorno. 
Figura 5.16 - Medidas do sinal de saída do transdutor com anel sensor na configuração horizontal, em função da posição da agulha, para sentidos opostos de movimentação da agulha. 103

Figura 5.17 - Esquema do experimento para avaliar a variação com a posição do pólo da agulha, com anel na horizontal. (a) sentido normal dos pólos, (b) pólos girados de $180^{\circ}$.

104

Figura 5.18 - Medidas do sinal de saída do transdutor com anel sensor na configuração horizontal, em função da posição da agulha, para sentidos opostos de posição do pólo da agulha.

104

Figura 5.19 - Esquema do experimento para analisar o comportamento do transdutor em função da direção do campo da Terra, com anel na configuração horizontal. (a) agulha perpendicular ao campo magnético terrestre, (b) agulha paralela ao campo magnético terrestre. 105

Figura 5.20 - Medidas do sinal de saída do transdutor com anel sensor na configuração horizontal, em função da posição da agulha, variando-se a direção relativa ao campo magnético da Terra.

106

Figura 5.21 - Variação do sinal de saída do transdutor com anel sensor na configuração horizontal, em função da posição da agulha, para distâncias entre o sensor e a agulha de $2 \mathrm{~cm}$ a $5 \mathrm{~cm}$.

107

Figura 5.22 - Amplitude máxima do sinal de saída em função da distância entre o sensor e a agulha, para o anel na configuração horizontal. 107 Figura 5.23 - Mapa do sinal de saída do transdutor para o anel na configuração vertical, com a distância entre o sensor e a agulha de $2 \mathrm{~cm}$, em relação à extremidade mais próxima do anel. Amplitude máxima = $0,5170 \mathrm{~V}$.

108

Figura 5.24 - Simulação feita com $\Delta V / \Delta H=12, m=0,35 \mathrm{Am}^{2}$. Amplitude máxima $=0,5577 \mathrm{~V}$.

109

Figura 5.25 - Mapa do sinal de saída do transdutor para o anel na configuração horizontal, com a distância entre o sensor e a agulha de $2 \mathrm{~cm}$. Amplitude máxima =0,697 V.

Figura 5.26 - Simulação feita com $\Delta V / \Delta H=12, m=0,28 \mathrm{Am}^{2}$. Amplitude máxima $=0,685 \mathrm{~V}$. 


\section{Lista de Abreviaturas}

SQUID - Superconducting Quantum Interference Device.

LTS - Low Temperature System.

HTS - High Temperature System.

MIG - Magnetoimpedância Gigante.

MRG - Magnetoresitência Gigante.

MIL - Magetoimpedância Longitudinal.

MIT - Magetoimpedância Transversal.

MIP - Magetoimpedância Perpendicular.

MPD - Movimento das Paredes dos Domínios.

SI - Sistema Internacional de Unidades.

CGS - Sistema de Unidades Gaussiano. 